\title{
Practical recommendations for treatment of hypertension in older patients
}

This article was published in the following Dove Press journal:

Vascular Health and Risk Management

28 June 2010

Number of times this article has been viewed

\section{Philip A Kithas \\ Mark A Supiano}

Geriatric Research, Education, and Clinical Center, George E Wahlen Department of Veterans Affairs Medical Center and Department of Internal Medicine, Division of Geriatrics, University of Utah School of Medicine, Salt Lake City, Utah, USA
Correspondence: Philip A Kithas

Geriatric Research, Education, and Clinical Center, Department of Veterans Affairs, Medical Center, 500 Foothill Drive, Salt Lake City, Utah 84I48, USA

Tel +l 80 I 5822455

Fax + I 80I 5845640

Email philip.kithas@va.gov
Background: By the year 2030 the percent of the population over the age of 65 years is projected to range from 3.7\% (in sub-Saharan Africa) to almost 22\% (in Europe). Accompanying this unprecedented growth will be a significant increase in many of the disease processes or "comorbidities" associated with aging, not the least of which is hypertension. Global health care resources and economies in general will be stressed to breaking point if this condition is not dealt with in an aggressive and timely manner because the consequences of untreated hypertension such as stroke, myocardial infarction, and dementia are exceedingly costly in the long term.

Methods: To help focus attention on the worldwide epidemic of hypertension, the current literature and guidelines were reviewed, along with information on the various classes of medications indicated in the treatment of hypertension in the elderly.

Results: Recent, large, randomized trials indicate that hypertension in the elderly can and should be treated to lower the incidence of stroke, myocardial infarction, and chronic kidney disease. Although thiazide-type diuretics are the recommended first-line agents in most cases of uncomplicated hypertension, multiple drug classes have been shown to be useful. In addition, and where feasible, a multidisciplinary team approach has demonstrated the most durable results.

Conclusion: Thiazide diuretics should be the first-line agents in uncomplicated, isolated systolic hypertension. Starting at low doses and proceeding in a gradual manner, these agents have proven efficacy in decreasing the risk of stroke and cardiovascular events. It is now recommended that these agents be used in low-dose combinations with other antihypertensive drug classes in patients who do not achieve target blood pressure $(<140 / 90 \mathrm{mmHg})$.

Keywords: isolated systolic hypertension, pulse pressure, ambulatory blood pressure monitoring

\section{Introduction}

Until recent studies provided evidence-based data showing that hypertension (HTN) in the elderly can and should be treated, and that multiple agents are effective, the prevailing opinion was that "the treatment of hypertension is a difficult and almost hopeless task". In fact, high blood pressure (BP) was believed to be a compensatory response to the narrowing of the coronary and cerebral vasculature that occurred with aging and as such should perhaps not even be "tampered" with.

Projections for the growth of the older US population estimate there will be 82 million persons over 65 years (20\% of the total population) and 19 million over 85 years ( $24 \%$ of the population over 65 years) by the year 2050 . This growth in the older population is a result not only of an increase in the overall population size but also a decline in several of the major causes of mortality. However, despite aggressive 
efforts directed at reducing the burden of cardiovascular disease, the age-related increase in BP combined with the increase in the aging population, portends a public health "tsunami".

Control of HTN in the elderly is imperative, not only to reduce the risk of cardiovascular disease, stroke, and chronic kidney disease, but also the incidence of atrial fibrillation, congestive heart failure (CHF), and cognitive impairment. Multiple antihypertensive agents have proven useful in the elderly. It has been shown that a $10 \mathrm{mmHg}$ reduction in systolic blood pressure (SBP) and a $5 \mathrm{mmHg}$ reduction in diastolic blood pressure (DBP) significantly decrease the incidence of myocardial infarction, stroke, $\mathrm{CHF}$, and overall mortality. Despite these findings, the rate of control of HTN is low, especially among the elderly, and reflects a need for more aggressive approaches, as well as improvements in the systems of care delivery. There is also a compelling need for research into the mechanisms of the age-related increase in $\mathrm{BP}$ and prevention of HTN in general.

\section{Classification}

Based on the current classification of HTN by the Joint National Committee on Prevention, Detection, Evaluation and Treatment of High Blood Pressure (JNC 7, Table 1) ${ }^{1}$ the estimated prevalence of HTN in the population over 65 years is $50 \%-75 \%$. For women over 75 years this value exceeds $75 \%$. In addition, data from the Framingham Heart Study show that over 20-25 years of follow-up, 85\% of men and women with normal BP at age 55 years will develop HTN by the time they attain the age of their average life expectancy. $^{2}$

Interestingly, the current classification of BP as set out by the JNC 7 makes no adjustment for age. JNC 7 incorporates research findings demonstrating that cardiovascular risk increases fairly linearly with increasing BP, and that this risk starts to increase above a BP level of $115 / 75 \mathrm{mmHg}$. In addition, there is now a category labeled "pre-hypertension", defined as

Table I JNC 7 classification of blood pressure levels ${ }^{\mathrm{a}}$

\begin{tabular}{llll}
\hline Category & $\begin{array}{l}\text { Systolic } \\
(\mathbf{m m H g})\end{array}$ & $\begin{array}{l}\text { Diastolic } \\
\mathbf{( m m H g )}\end{array}$ \\
\hline $\begin{array}{l}\text { Normal } \\
\text { Prehypertension }\end{array}$ & $120-139$ & and & $<80$ \\
$\begin{array}{l}\text { Hypertension } \\
\text { Stage 1 } \\
\text { Stage 2 }\end{array}$ & $140-159$ & or & $80-89$ \\
\hline
\end{tabular}

${ }^{a}$ Adapted from JNC 7 Express: The Seventh Report of the Joint National Committee on Prevention, Detection, Evaluation, and Treatment of High Blood Pressure. Bethesda, MD: National High Blood Pressure Education Program, National Heart, Lung and Blood Institute, National Institutes of Health, US Department of Health and Human Services; May 2003.'
SBP 120-139 mmHg or DBP 80-89 mmHg. Isolated systolic hypertension (ISH), the most common HTN subtype in the older population, has been removed as a distinct category. Instead, in almost all cases, HTN in the older population can be categorized on the SBP level alone.

\section{Age-related pathophysiologic changes}

It is highly unlikely that a single etiology is the explanation for essential HTN, no matter what a person's age. More probable is the contribution of multiple physiologic and lifestyle factors. Those factors likely contributing to age-related increase in BP include increased arterial stiffness (especially of the large arteries), ${ }^{3}$ decreased baroreceptor sensitivity, ${ }^{4}$ increased sympathetic nervous system activity, ${ }^{4}$ endothelial dysfunction, ${ }^{5}$ sodium sensitivity (decreased ability to excrete a sodium load), ${ }^{6}$ low plasma renin activity, insulin resistance, and the resulting effect on carbohydrate metabolism. ${ }^{7}$ Aldosterone may also be a significant contributor to HTN in later life. ${ }^{8}$ Lifestyle factors such as being sedentary, the presence of central adiposity, and a diet high in salt and fat are other contributors to HTN in the elderly.

Systolic HTN with an increased pulse pressure is the most commonly encountered form of HTN in the elderly and is associated with an increase in arterial stiffness. ${ }^{9}$ The degree of arterial stiffness has been shown to correlate with and predict the risk for adverse cardiovascular outcomes and overall mortality. ${ }^{10}$ Arterial stiffness can be determined noninvasively by techniques that measure pulse wave velocity between the carotid and femoral arteries. These measurements "span" the aorta and are therefore taken as a measure of aortic pulse wave velocity ${ }^{7}$ and stiffness. Recent studies have shown that despite comparable reductions in BP, antihypertensive agents from various classes have very different effects on aortic pulse wave velocity. ${ }^{11}$ This may explain why equivalent effects on lowering peripheral BP have not necessarily equated with improved clinical outcomes and why research is now focusing more on agents that result in reduced stiffness of the large arteries. Therefore, age-related changes in the reninangiotensin-aldosterone system are being actively studied.

Aldosterone is known to play several important roles mediated by both genomic and nongenomic pathways. Mineralocorticoid receptors are widely distributed, not only in the vasculature but also within the myocardium and central nervous system (CNS). In addition, evidence is accumulating that adipose tissue produces aldosterone secretory factors that promote excessive adrenal aldosterone production leading to insulin resistance, inflammation, oxidative stress, and 
sodium retention. ${ }^{8}$ Even within physiologic ranges, higher levels of plasma aldosterone have been shown to predispose individuals to developing HTN in the future. ${ }^{12}$

Other pathophysiologic changes characteristic in the elderly and important when contemplating treatment include the development of autonomic changes, such as decreased baroreceptor sensitivity and increased sympathetic nervous system activity. ${ }^{4}$ Under resting conditions, older individuals usually have no difficulty maintaining BP and adequate cerebral perfusion. However, with intravascular fluid shifts such as those occurring upon assuming an upright position or postprandially, or with exposure to vasodilator agents, the older individual is less able to compensate, and significant hypotension and decreased cerebral perfusion may occur. Due to baroreceptor insensitivity, larger changes in BP are required in order to initiate an appropriate compensatory increase in heart rate. In addition, the variability in BP increases with increasing age. The age-related increase in sympathetic nervous system activity may contribute to HTN through increased alpha-adrenergic receptor responsiveness. ${ }^{4}$

Other mechanisms involved in the increase in BP with aging include changes in the vascular endothelium resulting in increased vascular resistance. A decrease in the production of endothelial-derived nitric oxide impairs vasodilation and has been shown to correlate with aging and the development of HTN. ${ }^{5}$ A decrease in the kidney's ability to excrete a sodium load, related to decreases in renal blood flow and glomerular filtration, is another potential contributor to the observed increase in BP with aging. Salt sensitivity (defined as an increase in mean arterial $\mathrm{BP} \geq 5 \mathrm{mmHg}$ during a high versus a low sodium diet) is a finding in up to two-thirds of older hypertensive individuals.

\section{Evaluation and diagnosis}

Because BP is more variable in the elderly, HTN should never be diagnosed on the basis of a single measurement. At the initial visit, supine, sitting, and upright BPs should be determined and performed in both arms. The importance of a quiet environment (ideally using an automated instrument that obtains 5-6 measurements at 1-minute intervals with no medical personnel present in the room), sitting comfortably in a chair with the back supported and both feet flat on the floor, no intake of caffeine or use of tobacco for at least 30-45 minutes before measurement, appropriate cuff size, placement on bare skin, and resting with the arm supported at heart level for 5-10 minutes, cannot be overemphasized. Failure to follow these instructions can lead to significant, but false, elevations in BP. Three to five separate measurements should be made on each of 3 separate visits and the average calculated. Consideration must also be given to the existence of an auscultatory gap. Therefore, the maximum inflation pressure should be determined by palpating the radial or brachial pulse during cuff inflation and inflating well above the point where the pulse disappears. Determining the presence of postural hypotension, ie, a decrease of at least $20 \mathrm{mmHg}$ in the SBP or $10 \mathrm{mmHg}$ in the DBP in association with reported symptoms of dizziness or light-headedness within the first 2-5 minutes of assuming an upright posture, is also necessary in order to avoid increasing the risk of falls with the addition of antihypertensive medications.

Another consideration in evaluating HTN is the existence of office ("white-coat") HTN. This should be suspected when BP values determined in the clinic are consistently greater than those measured at home. In these cases, serious consideration should be given to either carefully obtained home values using an appropriately calibrated BP monitor or obtaining 24-hour ambulatory BP measurements. The technique of 24-hour ambulatory BP monitoring is useful for several reasons. ${ }^{13}$ Firstly, it defines daytime and nocturnal as well as the average BP over an entire 24-hour period. Target organ damage (eg, left ventricular hypertrophy [LVH], decreased renal function/microalbuminuria, carotid plaque, or hypertensive retinopathy) is best correlated with the 24-hour average value. Secondly, patients who fail to decrease their average nocturnal SBP by at least $10 \%$ of the average daytime SBP value (non-dippers) have been found to possess a more adverse cardiovascular risk profile compared with those who do drop by $>10 \%$ (dippers). ${ }^{14}$

In order to obtain reliable home values it is imperative that patients be educated in appropriate BP measurement techniques. As with office measurement, the patient needs to be sitting quietly for 5-10 minutes with his or her back supported in a chair with feet flat on the floor and arm supported at heart level. Morning and evening measurements should be made in triplicate, with the first discarded and the last two averaged. This should be done over a 7-day period, with the first 2 days discarded and the last 5 averaged. A similar technique can be used when/if treatment is initiated but with only once-a-day measurements taken just before administration of the majority of antihypertensive medication(s).

As with the younger population, greater than $90 \%$ of the elderly with high BP have essential HTN. With few exceptions, the same guidelines used in younger patients should be applied in the older population when secondary forms of HTN are suspected. Because ISH is the predominant form 
in the elderly, those who present with isolated diastolic HTN deserve careful consideration, with attention given to a renovascular etiology. Because of the propensity toward polypharmacy, the importance of a thorough medication review cannot be overemphasized. Attention should be paid to those medications known to increase BP, particularly corticosteroids and non-steroidal anti-inflammatory drugs, including the Cox-2 inhibitors. The high prevalence of obstructive sleep apnea in the elderly hypertensive population dictates this condition should be considered as a cause of high BP. Finally, although rare, the incidence of pheochromocytoma has been shown in autopsy studies to increase with age.

The evaluation of the older hypertensive patient is not complete without a thorough investigation of target organ damage, cardiovascular risk assessment, and identification of comorbid conditions that may impact the choice of antihypertensive therapy. The presence or absence of renal dysfunction, proteinuria/microalbuminuria, hypertensive retinopathy, and LVH should be determined. Cardiovascular risk should be assessed based on age, tobacco and/or alcohol use, lipid profile, salt and fat intake, and level of physical activity. Although somewhat controversial, factors consistent with the metabolic syndrome (impaired fasting glucose, HTN or its treatment, LDL $<40$ in men and $<50$ in women, waist circumference $>35$ inches in men and $>40$ inches in women, and hypertryglyceridemia) should also be determined and addressed.

\section{Treatment}

Data from multiple, randomized, placebo-controlled trials overwhelmingly support the treatment of HTN in the elderly and that this treatment is safe and effective. The SHEP (Systolic Hypertension in the Elderly Program) trial followed 4736 individuals (average age 72 years) with ISH (average baseline SBP $170 \mathrm{mmHg}$; average DBP $77 \mathrm{mmHg}$ ) for 4.5 years (average follow-up). Those randomized to active therapy (chlorthalidone $12.5 \mathrm{mg}$ up to $25 \mathrm{mg} /$ day, followed by atenolol $25 \mathrm{mg}$ up to $50 \mathrm{mg}$ /day if indicated) achieved an average SBP of $143 \mathrm{mmHg}$ compared with 155 $\mathrm{mmHg}$ in the placebo group. Active treatment reduced the incidence of total stroke by $36 \%$ and major cardiovascular events by $32 \%{ }^{15}$ The Medical Research Council (MRC) trial of treatment of HTN in older adults randomized 4396 patients aged 65-74 years from 226 general practices to treatment with a diuretic (hydrochlorothiazide $25 \mathrm{mg}$ or $50 \mathrm{mg}$ plus amiloride $2.5 \mathrm{mg}$ or $5 \mathrm{mg}$ daily), a beta-blocker (atenolol $50 \mathrm{mg}$ daily) or placebo. Mean SBP at baseline was 160-209 mmHg, mean DBP < $115 \mathrm{mmHg}$. After a mean follow-up of 5.8 years, active treatment (diuretic and betablocker groups combined) showed a $25 \%$ reduction in stroke, a $19 \%$ reduction in coronary events, and a $17 \%$ reduction in all cardiovascular events. The study also found that after adjustment for baseline characteristics, the diuretic group showed significant reductions in stroke $(31 \%)$, coronary events (44\%), and all cardiovascular events (35\%), whereas the beta-blocker group showed no significant reductions in the same endpoints. ${ }^{16}$ At a median follow-up of 2 years in patients over the age of 60 years with ISH (baseline SBP 160-219 mmHg, DBP < 95 mmHg), the Syst-Eur (Systolic Hypertension in Europe) trial demonstrated a $42 \%$ reduction in total stroke, $44 \%$ reduction in non-fatal stroke, and a $31 \%$ reduction in fatal/non-fatal cardiovascular endpoints in the active treatment group compared with those on placebo. Active treatment consisted of nitrendipine 10-40 $\mathrm{mg}$ /day with the possible addition of enalapril 5-20 mg/ day and/or hydrochlorothiazide $12.5-25 \mathrm{mg} / \mathrm{day}$, and this regimen achieved a $23 / 7 \mathrm{mmHg}$ reduction in SBP and DBP, respectively, compared with $13 / 2 \mathrm{mmHg}$ in the placebo group. ${ }^{17}$ Overall, significant reductions in mortality $(12 \%-$ $25 \%)$, stroke (35\%), and myocardial infarction (25\%), as well as chronic kidney disease (CKD) and CHF, have been demonstrated with active therapy. Put in terms of the number needed to treat (assuming a decrease in SBP of $12 \mathrm{mmHg}$ over 10 years), anywhere from 9 (highest-risk group) to 81 (lowest-risk group) patients would need to be treated to prevent one death.

Until completion of the recent Hypertension in the Very Elderly Trial (HYVET), few randomized controlled trials of HTN included subjects over the age of 80 years and fewer still included those over 85 years of age. HYVET randomized 3845 individuals over 80 years of age to either extendedrelease indapamide with the addition of perindopril (to achieve a target SBP $<150 \mathrm{mmHg}$ ) or placebo and was halted early when the data safety monitoring committee observed a $21 \%$ reduction in total mortality (absolute risk reduction $2.2 \%$, number needed to treat $=45$ over a median 1.8 -year period) in the treatment group. The intervention group also demonstrated significant reductions in fatal and non-fatal stroke, CHF, and cardiovascular events. ${ }^{18}$ HYVET subjects were generally healthy, community-dwelling older adults with a standing BP of $>140 \mathrm{mmHg}$; those with dementia, nursing home residents, and those with an inability to walk were excluded. Therefore, it is important to note that results cannot be extrapolated to the frail elderly. In addition, a substudy of HYVET (HYVET-COG) that assessed cognitive function and the rate of development of dementia showed 
a similar incidence of dementia between intervention and control groups.

Having demonstrated that HTN in the elderly can and should be treated, it is important to consider general geriatric principles and individualize patient goals. The risk of the most common treatment-related side effect, ie, postural hypotension, must be balanced against any potential benefits of treatment. In general, SBP should remain the primary target of therapy because it is a stronger predictor of adverse outcomes than is DBP. A SBP of 135-140 mmHg (SBP < $130 \mathrm{mmHg}$ in type 2 diabetes or CKD) should be the treatment goal, utilizing agents least likely to negatively impact functional status and quality of life. For example, if a patient's seated SBP cannot be lowered to $<140 \mathrm{mmHg}$ without inducing significant orthostasis, then a focus on standing SBP may be a more prudent approach. In cases of a markedly elevated SBP, an initial goal of $<160 \mathrm{mmHg}$ is reasonable, provided there is no evidence of target organ damage, or signs or symptoms of a hypertensive emergency.

Concerns have been raised about lowering BP below a certain threshold and the potential for adverse outcomes. Some studies have shown increased mortality, particularly with regard to DBP, below a certain threshold (the J-shaped curve). Post hoc analysis of the Systolic Hypertension in the Elderly trial ${ }^{19}$ suggested that DBP $<70 \mathrm{mmHg}$ was associated with more cardiovascular events, but only in those with a history of coronary artery disease (CAD). Diastolic BP as low as $55 \mathrm{mmHg}$ was not associated with increased cardiovascular mortality, but noncardiovascular mortality was increased (hazard ratios were higher). It is therefore prudent to avoid aggressive lowering of DBP (eg, $<70 \mathrm{mmHg}$ ), especially in individuals with CAD. Diastolic BP lower than this value is related to increased pulse pressure which, in turn, reflects increased arterial stiffness. Subsequent decreases in endocardial perfusion would then lead to tissue ischemia and an expected increased risk of adverse coronary and cerebrovascular events.

\section{Nonpharmacologic approaches}

Nonpharmacologic or lifestyle interventions can have significant BP lowering effects and remain important adjuncts to drug therapy. In addition to potentiation of pharmacologic therapy, a decrease in other cardiovascular risk factors may be observed. Because the typical physiologic profile of the older hypertensive individual includes obesity, salt sensitivity, and lack of exercise, interventions directed at these characteristics can be expected to have a significant impact (Table 2). Weight reduction, aerobic exercise, and a decrease in sodium and alcohol intake can each result in up to a $10 \mathrm{mmHg}$
Table 2 Nonpharmacologic therapies in stage I hypertension

- Weight loss with a focus on reduction of central adiposity

- Aerobic exercise (goal 30 minutes/day, 5 days/week) combined with strength training

- Smoking cessation

- Moderation of alcohol intake (two drinks per day in men, one in women)

- Modification of diet to decrease sodium, cholesterol, and saturated fat intake while maintaining adequate potassium, magnesium, and calcium intake

decrease in SBP which is equivalent to that achieved with monotherapy. Dietary sodium restriction (100-200 mEq/day) and weight loss in older hypertensive patients were studied in the Trial of Non-Pharmacologic Intervention in the Elderly. ${ }^{20}$ The intervention group achieved an average $40 \mathrm{mmol} /$ day reduction in sodium intake and an average $4 \mathrm{~kg}$ weight loss which were associated with a $30 \%$ decrease in the need to reinitiate antihypertensive therapy. In general, in elderly Stage 1 hypertensive patients without diabetes, a 6-month trial of nonpharmacologic interventions is warranted as the first step in the treatment algorithm.

\section{Pharmacologic interventions}

In addition to the nonpharmacologic interventions discussed, a general approach to antihypertensive therapy includes the use of agents based on a patient's comorbidities (eg, diabetes, CHF, CAD), a low-dose diuretic in uncomplicated HTN, starting at half the usual dosage and increasing slowly, a focus on SBP, avoiding excessive lowering of DBP, and accounting for individual patient goals (Table 3). Racial and ethnic background must also be taken into consideration. Given these considerations, the initial agent in simple, uncomplicated Stage 1 HTN will usually be a low-dose thiazide-type diuretic. In patients with a SBP $>20 \mathrm{mmHg}$ above their target, it is likely that 2 drugs will need to be initiated. Current recommendations suggest combinations of a low-dose thiazide diuretic with an

Table 3 General approach to pharmacologic therapy

- Base therapy on pre-existing comorbidities, eg, diabetes, heart failure,CAD

- Begin with a low-dose diuretic in uncomplicated hypertension

- Begin therapy at half the usual dose and increase slowly, consider low-dose combination therapy if goal SBP is not met on single-agent therapy

- Focus on systolic blood pressure and patient comorbidities

- Avoid excessive lowering of diastolic blood pressure ( $<70 \mathrm{mmHg}$ )

- Adjust goals when adverse events (eg, postural hypotension, postprandial hypotension) cannot be avoided

- Continue and emphasize nonpharmacologic therapies throughout treatment

Abbreviations: CAD, coronary artery disease; SBP, systolic blood pressure. 
angiotensin-converting enzyme inhibitor (ACEI), angiotensin receptor blocker (ARB), or long-acting calcium channel antagonist (CCA). Many preparations are available combined into a single tablet which would improve ease of administration and compliance. More recently, evidence is beginning to demonstrate that $\mathrm{BP}$ reductions may be greater and side effects lower with low-dose combinations of 2 or more antihypertensive agents than with a single agent increased to full dose. As there is no universal agreement on the approach to choosing alternative agents or combination therapy, these decisions should be based on a patient's comorbidities, weighted with the advantages and disadvantages of a specific drug. In general, centrally acting agents (eg, clonidine, methyldopa) and direct vasodilators (minoxidil), due to their CNS side effects (sedation) and propensity to cause significant orthostatic hypotension, should be avoided. Finally, beta-blockers are best avoided in the older hypertensive patient without a specific indication, such as CAD. In the older population, beta-blockers have been associated with no reduction in the risk of stroke or coronary events. ${ }^{13}$

\section{Thiazide-type diuretics}

Several properties of the thiazide-type diuretics have led to them being recommended as first-line therapy in most older adults with uncomplicated Stage 1 HTN. At low doses ( $<25 \mathrm{mg}$ /day of hydrochlorothiazide or equivalent) these agents have been demonstrated in randomized controlled trials to reduce mortality, stroke, and cardiovascular events in the older hypertensive population. ${ }^{21}$ In addition, they have fewer side effects of hypokalemia, hyperuricemia, hypomagnesemia, hypertriglyceridemia, and glucose intolerance when used at recommended doses, with low cost and a wide therapeutic index, along with once-daily dosing. There is good synergy with agents of different classes (eg, ACEIs, ARBs, and CCAs) and most importantly in the elderly, these drugs preferentially lower SBP relative to DBP. Finally, it is important to note that maintaining potassium levels is key in order to reduce the potential for arrhythmias, because higher doses have been shown to increase the incidence of sudden cardiac death, ${ }^{22}$ as well as impairment of glucose tolerance. Also, this class is often ineffective at serum creatinine levels $>2 \mathrm{mg} / \mathrm{dL}$ or creatinine clearance below $30-40 \mathrm{~mL} / \mathrm{min}$.

\section{Angiotensin-converting enzyme inhibitors}

This class of medication (eg, lisinopril 10-40 mg/day or equivalent), shown to be effective in the lowering of BP in the elderly, is also generally well tolerated and represents an acceptable alternative first-line therapy or in combination with a thiazide-type diuretic. These agents possess a favorable safety profile and lack significant CNS or metabolic side effects. In particular, the ACEIs have shown significant benefits in patients with diabetes (particularly with coexistent microalbuminuria) and those with left ventricular systolic dysfunction. In several clinical conditions, this class is associated with preservation of renal function. Generalized side effects include cough, angioedema, hyperkalemia (particularly in the setting of renal insufficiency or in combination with nonsteroidal anti-inflammatory drugs or potassiumsparing diuretics), and acute renal failure in patients with significant bilateral renal artery stenosis. These agents are contraindicated in pregnancy.

\section{Angiotensin receptor blockers}

To date, there are no randomized controlled trials comparing ARBs with diuretics and their effects on treatment outcomes in the elderly. A single meta-analysis has suggested that therapy with diuretics is superior to that with ARBs for most outcomes. As a result, this class is not generally recommended as first-line therapy in uncomplicated HTN. Rather, they should be considered in those patients with diabetes, CHF, or chronic kidney disease who are intolerant of ACEIs. Angioedema is considered rare with ARBs and they are not a cause of cough.

\section{Calcium channel antagonists}

Although all three chemical classes of CCAs have shown effectiveness in lowering BP in the older hypertensive patient, it is the long-acting dihydropyridine class that has been the most widely studied. Significant reductions in stroke risk in older hypertensive patients were demonstrated in the Systolic Hypertension in Europe ${ }^{23}$ and China Trials. ${ }^{24}$ Results from patients with very high cardiac risk enrolled in the Avoiding Cardiovascular Events through Combination Therapy in Patients Living with Systolic Hypertension (ACCOMPLISH) trial demonstrated the superiority of an ACEI-CCA (amlodipine) combination over an ACE-thiazide combination with regard to a decrease in cardiovascular events despite comparable BP-lowering effects. ${ }^{25}$ As a result of age-related pharmacokinetic changes (decreased clearance and increased plasma levels), lower CCA dosages should be used in the elderly. It should be emphasized that the longacting formulations are preferred, and that there is no role for short-acting CCAs in the treatment of HTN due to their propensity to lower SBP rapidly. Side effects include headache, lower extremity edema, gingival hyperplasia, worsening of 
gastroesophageal reflux symptoms due to relaxation of the lower esophageal sphincter, and constipation.

\section{Beta receptor antagonists}

Review of data from randomized controlled trials has questioned the efficacy of beta-receptor antagonists in the treatment of older individuals with HTN. These agents appear to be less effective than low-dose thiazide diuretics with regard to improving BP and cardiovascular outcomes as initial therapy, and are more likely to be discontinued due to adverse side effects. As a result, this class is designated as a second-line therapy by JNC 7 and should not be considered as first-line in uncomplicated HTN in the elderly. Clearly, these agents are indicated in the older HTN population with certain comorbidities, such as symptomatic CAD, in secondary prevention after myocardial infarction, and in some patients with systolic dysfunction.

\section{Alpha receptor antagonists}

Although this class has been demonstrated to reduce peripheral vascular resistance and lower BP, the development of symptomatic postural hypotension has severely limited their utility, particularly in the elderly. In addition, data from the Antihypertensive and Lipid-Lowering Treatment to Prevent Heart Attack Trial (ALLHAT) showed that subjects randomized to doxazosin had a 2-fold higher risk of hospitalization for CHF compared with those randomized to the diuretic arm. ${ }^{21}$ These medications may be considered as additional therapy, particularly in older hypertensive men with benign prostatic hypertrophy.

\section{Aldosterone antagonists}

Initially developed as therapy for cirrhosis and primary hyperaldosteronism, and later as additional agents in the treatment of $\mathrm{CHF}$, aldosterone antagonists may prove useful as first-line agents in the elderly with uncomplicated HTN. As with thiazide-type diuretics, the aldosterone antagonists preferentially lower SBP. In addition, this class antagonizes aldosterone's metabolic effects of increasing sodium resorption, altering renal hemodynamics, and increasing afterload and vascular stiffness, all of which are important pathophysiologic characteristics of geriatric HTN.

\section{Follow-up visits}

Follow-up visit frequency is based on the level of HTN at presentation and for most patients (except those with SBP $>180 \mathrm{mmHg}$ ), a visit frequency of once every 1-2 months for dosage adjustment is appropriate. Achieving target BP levels rapidly (except in cases of hypertensive emergencies) is unnecessary and should be avoided because overtreatment may result in decreased cerebral and coronary perfusion, and subsequent postural or postprandial hypotension. At each visit, both supine and standing BPs should be determined. Adjustments to therapy may need to be based on standing values if postural hypotension is present. Before instituting an increase in dosage or an additional agent, the patient's adherence to therapy should be thoroughly assessed. The use of medications that can worsen BP control (eg, nonsteroidal anti-inflammatory drugs, including Cox-2 agents, and corticosteroids) should also be reviewed.

If, after performing the above review, a patient's BP is still not at target, then a careful increase in dosage, addition of another agent (thiazide if not already being used and renal function is appropriate with a calculated creatinine clearance of $>30-40 \mathrm{~mL} / \mathrm{min}$ ), or switching to another class of medication can be considered. Continuing efforts at lifestyle modifications should be re-emphasized, along with counseling that several months may be needed to achieve the target value. In general, target BPs are the same as those in a younger population, and are outlined in JNC 7. Provided there is no evidence for a significant orthostatic drop in SBP, a goal of $<140 / 90 \mathrm{mmHg}$ is reasonable. In patients with diabetes mellitus and/or CKD the goal becomes $<130 / 80 \mathrm{mmHg}$.

As with the younger hypertensive population, if target $\mathrm{BP}$ is not achieved on a 3-drug regimen with documented compliance, then an evaluation for resistant or secondary HTN (renovascular, primary hyperaldosteronism, obstructive sleep apnea, renal artery stenosis, pheochromocytoma, Cushing's) should be undertaken. Finally, after more than a year of stable BP control at target levels, consideration can be given to a step-down in therapy, especially in patients who have been successful in lifestyle modifications. Nevertheless, dosages should be decreased slowly and cautiously while maintaining close BP monitoring.

\section{Hypertensive emergencies and urgencies}

By definition, a hypertensive emergency involves an elevated BP (usually $>180 \mathrm{mmHg}$ systolic) in association with signs and/or symptoms of target organ damage. The resultant vascular compromise of the affected organ may include hypertensive encephalopathy, acute heart failure with pulmonary edema (flash pulmonary edema), dissecting aortic aneurysm, acute renal failure, and unstable angina. Management requires acute hospitalization for administration of parenteral 
antihypertensives, with continuous BP monitoring to lower BP rapidly yet not initially to a normal level. Normalization of BP immediately can lead to coronary and cerebral hypoperfusion syndromes. Therefore, BP should be lowered no more than $25 \%$ in the first 2 hours, with gradual lowering over the first 6 hours to a target of less than 160/100 mmHg.

In comparison with emergencies, hypertensive urgencies are situations requiring BP lowering within 24 hours in order to avoid target organ damage. Again, gradual lowering of BP is indicated, and can be obtained by administering oral agents in the setting of close follow-up.

\section{Conclusion}

In order to avoid the aforementioned hypertensive "tsunami" and its subsequent cost to patients, and the general public and health care system overall, several barriers to achieving adequate BP control need to be addressed and overcome. The older hypertensive population with its higher incidence of multiple comorbidities requires an aggressive approach to choice of therapeutic regimen(s), intensity of therapy, consideration of the increased incidence of resistant HTN and ultimately, the goals of care (ie, which treatment guidelines will take precedence in a given patient's circumstances). Likewise, the fact that there is no cure for this chronic condition and that it is essentially "asymptomatic", engenders poor adherence to recommended lifestyle changes and pharmacologic therapy. Additional obstacles to achieving recommended BP levels include poor health literacy, low financial status/cost of medications, poor or no health insurance coverage, lack of adequate access to care and frequency of follow-up needed based on BP levels, and physicians' overestimation of their own compliance with guidelines and achievement of BP goals.

With the goal of improving BP control in the burgeoning, older, hypertensive population, several quality improvement strategies have proven useful. Of these, a combination of a multidisciplinary approach, home and ambulatory BP monitoring, and patient education have proven the most effective. In this context, an interdisciplinary geriatric team is well suited to address all of these areas. Nurses trained in the management of HTN can provide feedback on the level of BP control, dieticians can review dietary compliance and provide education and reinforcement, pharmacists can evaluate for medication side effects/interactions and promote adherence, and social workers may be able to offer assistance with the financial burdens of obtaining and continuing medication. For those patients who are housebound, advanced practice nurses or physician assistants with physician support, can provide many of the same services as part of a similar multidisciplinary "home-based primary care" team. Employing these strategies should more than adequately address the most important factors in improving BP control, ie, frequent monitoring and follow-up measures (compliance, education, maintaining long-term control of BP, and evaluation for adverse effects of treatment).

In summary, given the above conditions, the following general therapeutic recommendations apply in the elderly population:

- Diuretics, starting at low doses and increased gradually, should be first-line in those patients without comorbid conditions.

- In patients with diabetes mellitus (particularly those with proteinuria) the ACEIs (ARBs if there is cough with an ACEI) are considered first-line.

- In those with systolic heart failure, a multi-pronged approach using ACEIs, diuretics (thiazide or loop, depending on renal function) and aldosterone antagonists is indicated.

- The presence of CAD is an indication for the use of betareceptor antagonists (without intrinsic sympathomimetic activity).

- Renal insufficiency with proteinuria from any cause should be managed with ACEIs, ARBs, and CCAs of the nondihydropyridine class.

\section{Disclosure}

The authors report no conflict of interest in this work.

\section{References}

1. Chobanian AV, Bakris GL, Black HR, et al. The Seventh Report of the Joint National Committee on Prevention, Detection, Evaluation, and Treatment of High Blood Pressure. Hypertension. 2003;42:1206-1252.

2. Vasan RS, Beiser A, Seshadri S, et al. Residual lifetime risk for developing hypertension in middle-aged women and men: The Framingham heart study. JAMA. 2002;287:1003-1010.

3. Lakatta EG. Central arterial aging and the epidemic of systolic hypertension and atherosclerosis. J Am Soc Hypertens. 2007;1:302-340.

4. Supiano MA, Hogikyan RV, Sidani MA, et al. Sympathetic nervous system activity and alpha-adrenergic responsiveness in older hypertensive humans. Am J Physiol. 1999;276:E519-E528.

5. Celermajer DS, Sorensen KE, Spiegelhalter DJ, et al. Aging is associated with endothelial dysfunction in healthy men years before the age-related decline in women. J Am Coll Cardiol. 1994;24:471-476.

6. Weinberger MH. Sodium and blood pressure 2003. Curr Opin Cardiol. 2004; 19:353-356.

7. Sengstock DM, Vaitkevicius PV, Supiano MA. Arterial stiffness is related to insulin resistance in nondiabetic hypertensive older adults. J Clin Endocrinol Metab. 2005;90:2823-2827.

8. Sowers JR, Whaley-Connell A, Epstein M. Narrative Review: The emerging clinical implications of the role of aldosterone in the metabolic syndrome and resistant hypertension. Ann Int Med. 2009;150:776-783.

9. Chobanian AV. Isolated systolic hypertension in the elderly. $N$ Engl $J$ Med. 2007;357:789-796. 
10. Boutouyrie P, Tropeano AI, Asmar R et al. Aortic stiffness is an independent predictor of primary coronary events in hypertensive patients. A longitudinal study. Hypertension. 2002;39:10-15.

11. Mackenzie IS, McEniery CM, Dhakam Z, et al. Comparison of the effects of antihypertensive agents on central blood pressure and arterial stiffness in isolated systolic hypertension. Hypertension. 2009;54:409-413.

12. Vasan RS, Evans JC, Larson MG, et al. Serum aldosterone and the incidence of hypertension in nonhypertensive persons. $N$ Engl J Med. 2004;351:33-41.

13. Clement DL, De Buyzere ML, De Bacquer Da, et al. Prognostic value of ambulatory blood-pressure recordings in patients with treated hypertension. N Engl J Med. 2003;348:2407-2415.

14. Ohkubo T, Hozawa A, Yamaguchi J, et al. Prognostic significance of the nocturnal decline in blood pressure in individuals with and without high 24-h blood pressure: The Ohasama study. J Hypertension. 2002;20:2183-2189.

15. SHEP Cooperative Research Group. Prevention of stroke by antihypertensive drug treatment in older persons with isolated systolic hypertension. Final results of the Systolic Hypertension in the Elderly Program (SHEP). JAMA. 1991;265:3255-3264.

16. Medical Research Council Working Party. MRC trial of treatment of hypertension in older adults: rincipal results. BMJ. 1992;304:405-412.

17. Staessen JA, Fagard R, Lutgarde T, et al; for the Systolic Hypertension in Europe (Syst-Eur) Trial Investigators. Randomised double-blind comparison of placebo and active treatment for older patients with isolated systolic hypertension. Lancet. 1997;350:757-764.

18. Beckett NS, Peters R, Fletcher A, et al. Treatment of hypertension in patients 80 years of age or older. N Engl J Med. 2008;358:1887-1898.
19. Somes GW, Pahor M, Shorr RI, et al. The role of diastolic blood pressure when treating isolated systolic hypertension. Arch Intern Med. 1999; 159:2004-2009.

20. Whelton PK, Appel LJ, Espeland MA, et al. Sodium reduction and weight loss in the treatment of hypertension in older persons: A randomized controlled trial of non-pharmacologic interventions in the elderly (TONE). JAMA. 1998;279:839-846.

21. ALLHAT Officers and Coordinators for the ALLHAT Collaborative Research Group. Major outcomes in high-risk hypertensive patients randomized to angiotensin-converting enzyme inhibitor or calcium channel blocker vs diuretic: The Antihypertensive and Lipid-Lowering Treatment to Prevent Heart Attack Trial (ALLHAT). JAMA. 2002;288:2981-2997.

22. Siscovick DS, Raghunathan TE, Psaty BM, et al. Diuretic therapy for hypertension and the risk of primary cardiac arrest. $N$ Engl J Med. 1994;330:1852-1857.

23. Staessen JA, Fagard R, Thiis L, et al. Randomised double-blind comparison of placebo and active treatment for older patients with isolated systolic hypertension. Lancet. 1997;350:757-764.

24. Ji-Guang W, Staessen JA, Lansheng G, et al. Chinese trial on isolated systolic hypertension in the elderly. Arch Intern Med. 2000;160: $211-220$.

25. Jamerson K, Weber MA, Bakris GL, et al. Benazepril plus amlodipine or hydrochlorothiazide for hypertension in high-risk patients. $N$ Engl J Med. 2008;359:2417-2428.
Vascular Health and Risk Management

\section{Publish your work in this journal}

Vascular Health and Risk Management is an international, peerreviewed journal of therapeutics and risk management, focusing on concise rapid reporting of clinical studies on the processes involved in the maintenance of vascular health; the monitoring, prevention and treatment of vascular disease and its sequelae; and the involvement of

\section{Dovepress}

metabolic disorders, particularly diabetes. This journal is indexed on PubMed Central and MedLine. The manuscript management system is completely online and includes a very quick and fair peer-review system, which is all easy to use. Visit http://www.dovepress.com/ testimonials.php to read real quotes from published authors. 